Research in Astron. Astrophys. 2009 Vol. 9 No. 1, 5 - 10

http://www.raa-journal.org http://www.iop.org/journals/raa

$\boldsymbol{R}$ esearch in

Astronomy and

Astrophysics

\title{
LETTERS
}

\section{Discovery of four gravitational lensing systems by clusters in the SDSS DR6 *}

\author{
Zhong-Lue Wen ${ }^{1}$, Jin-Lin $\mathrm{Han}^{1}$, Xiang-Yang Xu ${ }^{1,2}$, Yun-Ying Jiang ${ }^{1}$, Zhi-Qing Guo ${ }^{1}$, \\ Peng-Fei Wang ${ }^{1}$ and Feng-Shan Liu ${ }^{1,3}$ \\ 1 National Astronomical Observatories, Chinese Academy of Sciences, Beijing 100012, China; \\ zhonglue@bao.ac.cn,hjl@bao.ac.cn \\ 2 College of Science, Guizhou University, Guiyang 550025, China \\ ${ }^{3}$ College of Physics Science and Technology, Shenyang Normal University, Shenyang 110034, China
}

\begin{abstract}
We report the discovery of 4 strong gravitational lensing systems by visual inspections of the Sloan Digital Sky Survey images of galaxy clusters in Data Release 6 (SDSS DR6). Two of the four systems show Einstein rings while the others show tangential giant arcs. These arcs or rings have large angular separations $\left(>8^{\prime \prime}\right)$ from the bright central galaxies and show bluer color compared with the red cluster galaxies. In addition, we found 5 probable and 4 possible lenses by galaxy clusters.
\end{abstract}

Key words: galaxies: clusters: general — gravitational lensing

\section{INTRODUCTION}

The strong gravitational lensing by galaxy clusters not only constrains their mass distributions (e.g. Takahashi \& Chiba 2001, Shu et al. 2008) but also provides an independent way to study highredshift faint background galaxies, which are otherwise unobservable (e.g. Miralda-Escude \& Fort 1993, Metcalfe et al. 2003). Furthermore, the statistics of gravitational arcs in lensing clusters can be used to constrain the paradigm of structure formation and cosmology (e.g. Bartelmann et al. 1998, Li et al. 2005).

Up to now, about 120 strong lensing clusters have been discovered (e.g. Lynds \& Petrosian 1989, Smail et al. 1991, Luppino et al. 1999, Zaritsky \& Gonzalez 2003, Gladders et al. 2003, Sand et al. 2005, Hennawi et al. 2008). Almost all previous searches for the lensing systems are based on the ground-based telescopes with seeing between $0.5^{\prime \prime}$ and $1.5^{\prime \prime}$. Sand et al. (2005) used the HST WFPC2 data and found 104 giant arcs in 54 clusters. Recently, based on 240 rich clusters identified from the Sloan Digital Sky Survey (SDSS, York et al. 2000) and the follow-up deep imaging observations from the Wisconsin-Indiana-Yale NOAO $3.5 \mathrm{~m}$ telescope and the University of Hawaii 88 inch telescope, Hennawi et al. (2008) uncovered 16 new lensing clusters with giant arcs, with 12 likely lensing clusters and 9 possible candidates.

In this letter, we report the discovery of 4 strong gravitational lensing systems by clusters in the SDSS DR6. The Einstein rings or giant arcs we found from the SDSS images suggest that they are excellent strong lensing systems. In addition, we found 5 probable and 4 possible lensing systems by galaxy clusters.

\footnotetext{
* Supported by the National Natural Science Foundation of China.
} 


\section{LENSING SYSTEMS DISCOVERED FROM THE SDSS DR6 IMAGES}

The SDSS provides five broad bands $(u, g, r, i$, and $z)$ for photometry and follow-up spectroscopy. The star-galaxy separation is reliable to the limit of $r=21.5$ (Lupton et al. 2001). The photometric data reaches the limit of $r=22.5$, with a mean seeing of $1.43^{\prime \prime}$ in $r$-band (Stoughton et al. 2002).

Several valuable lenses have been previously discovered in the SDSS data, though the survey is shallow (55 s exposure with a $2.5 \mathrm{~m}$ telescope) making it difficult to detect faint giant arcs. Allam et al. (2007) have serendipitously found the " 8 O'clock arc" from a Lyman break galaxy at $z=2.73$ lensed by a luminous red galaxy SDSS J002240.91+143110.4 at $z=0.38$. Belokurov et al. (2007; 2009) looked for multiple blue objects around luminous red galaxies, and discovered the "Cosmic horseshoe", an almost complete Einstein ring of diameter $10^{\prime \prime}$ around SDSS J114833.15+193003.5 at $z=0.444$, and also the "Cheshire Cat", which consists of two galaxies at $z=0.97$ and $z>1.4$ lensed by a combination of two giant early galaxies, SDSS J103847.95+484917.9 and SDSS J103839.20+484920.3 at $z=0.426$ and 0.432 , respectively. The lenses in the above systems are galaxies. Because there are a large number of high-redshift $(z \geq 0.3)$ clusters in the SDSS, they can act as efficient lenses. Estrada et al. (2007) preformed a systematic search for giant arcs in 825 SDSS maxBCG clusters (Koester et al. 2007) with masses $M \geq 2 \times 10^{14} M_{\odot}$, and found no gravitational arcs. However, they published a serendipitous discovery of the Hall's arc lensed by a cluster, SDSS J014656.0-092952 at $z=0.447$. Ofek et al. (2008) found the giant arc of a galaxy at $z=1.018$ lensed by a massive cluster SDSS J120923.7+264047 at $z=0.558$. Lin et al. (2008) found a galaxy at $z=2.00$ lensed by SDSS J120602.09+514229.5, the brightest galaxy in a cluster. Shin et al. (2008) found a post-starburst galaxy at $z=0.766$ lensed by a cluster galaxy at $z=0.349$.

We searched for the lensing systems by visual inspections of the color images of a large sample of clusters. We first searched for clusters by determining the luminous cluster members $\left(M_{r} \leq-21\right)$ using the photometric redshifts of galaxies brighter than $r=21.5$ (Csabai et al. 2003). A cluster at $z$ is identified when the number of member galaxies reaches 8 within a projected radius of $0.5 \mathrm{Mpc}$ and a photometric redshift ranges between $z \pm \Delta z$. Here, we set $\Delta z=0.04(1+z)$ to allow variable uncertainties of photometric redshifts at different redshifts. These criteria can significantly reduce the false detection rate of clusters. From the SDSS DR6, we identified $\sim 40,000$ clusters of $0.05<z<0.6$ with an overdensity greater than 4.5 (Wen et al. in preparation). Secondly, we searched for gravitational lensing features in these clusters by inspecting the composite ( $g, r$ and $i$ ) color images from the SDSS web page 1 independently by at least by three authors. We found 13 new candidates for lensing systems, in addition to the known cases in 6 clusters, as listed in Table 1 . In addition to the name, redshift and the mass of cluster, we give the angular separation of the arc from the bright central galaxy, the magnitude and color of the brightest part of the arc and notes in Table 1

The cluster mass, $M_{200}$, was estimated from the summed $r$-band luminosity within $r_{200}$. Here, $r_{200}$ is the radius within which the mean mass density is 200 times that of the critical cosmic mass density. The mass-to-light ratio was calibrated by comparing the cluster masses derived by Reiprich \& Böhringer (2002) with the summed $r$-band luminosities of a sample of clusters (Wen et al. in preparation). Such determined masses are the lower limits for clusters at $z>0.42$, because their members are incomplete at the faint end of $M_{r}=-21$.

By careful inspections of the SDSS images of these candidates, we almost certain found that 4 of them are lensing systems (see Fig. 1) though the images are somewhat shallow. For the system of SDSS J090002.6+223404, the three arclets (A, B, C) around the two central galaxies nearly form a circle with a radius of $8.4^{\prime \prime}$, which may be an Einstein ring if the arclets are the images of the same background source. SDSS J223831.3+131955 shows a faint Einstein ring with a radius of 9.3" . SDSS J111310.6+235639 and SDSS J134332.8+415503 show tangential giant arcs with respect to the bright central galaxies with a separation of more than $12^{\prime \prime}$. The faint but clear arcs usually have blue color compared with the bright central galaxies.

\footnotetext{
1 http://cas.sdss.org/astro/en/
} 
Table 1 Lensing systems found to be associated with the SDSS clusters.

\begin{tabular}{lllllll}
\hline Cluster name & $z$ & $\begin{array}{l}\mathrm{M}_{200} \\
\left(10^{14} M_{\odot}\right)\end{array}$ & $\begin{array}{l}\theta \\
\left({ }^{\prime \prime}\right)\end{array}$ & $r$ & $g-r$ & Reference, Notes \\
\hline SDSS J014656.0-092952 & 0.447 & $>41.0$ & 13.6 & $21.13 \pm 0.09$ & $0.54 \pm 0.13$ & 1, three giant arcs \\
NSC J082722.2+223244 & 0.335 & 23.8 & 4.4 & $20.16 \pm 0.05$ & $-0.13 \pm 0.06$ & 2, multiple images \\
MACS J113313.1+500840 & 0.389 & 11.6 & 10.0 & $21.62 \pm 0.13$ & $-0.10 \pm 0.16$ & 3 , giant arc \\
SDSS J120602.0+514229 & 0.442 & $>7.7$ & 4.3 & $20.29 \pm 0.05$ & $0.21 \pm 0.06$ & 4 , arc near galaxy \\
SDSS J120923.6+264046 & 0.558 & $>28.7$ & 11.3 & $22.37 \pm 0.26$ & $0.82 \pm 0.42$ & 5 , giant arc \\
NSCS J124034.0+450923 & 0.278 & 5.3 & 3.1 & $19.89 \pm 0.03$ & $-0.10 \pm 0.04$ & 6 , arc near galaxy \\
SDSS J090002.6+223404 & 0.489 & $>6.6$ & 8.4 & $20.41 \pm 0.06$ & $0.06 \pm 0.07$ & $7 *$, almost certain:Einstein ring? \\
SDSS J111310.6+235639 & 0.324 & 26.8 & 12.8 & $21.61 \pm 0.11$ & $1.53 \pm 0.28$ & 7 , almost certain:giant arc \\
SDSS J134332.8+415503 & 0.418 & 11.7 & 12.4 & $21.09 \pm 0.12$ & $0.10 \pm 0.16$ & 7 , almost certain:giant arc \\
SDSS J223831.3+131955 & 0.413 & 9.7 & 9.3 & $21.73 \pm 0.16$ & $0.57 \pm 0.24$ & $7 *$, almost certain:Einstein ring \\
SDSS J095739.1+050931 & 0.442 & $>5.8$ & 8.0 & $20.31 \pm 0.07$ & $0.18 \pm 0.09$ & 7 , probable:giant arc \\
SDSS J113740.0+493635 & 0.448 & $>5.6$ & 3.8 & $20.39 \pm 0.04$ & $0.04 \pm 0.05$ & $6,7 *$ probable:arc near galaxy \\
maxBCG J120735.9+525459 & 0.278 & 7.0 & 11.3 & $20.80 \pm 0.07$ & $1.21 \pm 0.15$ & 7 , probable:giant arc \\
SDSS J131811.5+394226 & 0.475 & $>6.6$ & 8.9 & $20.55 \pm 0.09$ & $0.44 \pm 0.13$ & 7 , probable:giant arc \\
NSCS J122648.0+215157 & 0.418 & 46.2 & 10.8 & $21.74 \pm 0.19$ & $0.17 \pm 0.22$ & 7 , probable:giant arc \\
SDSS J123736.2+553342 & 0.410 & 13.4 & 4.6 & $20.14 \pm 0.04$ & $0.18 \pm 0.05$ & 6,7*, possible:blue arc? \\
SDSS J131534.2+233301 & 0.517 & $>5.7$ & 5.5 & $20.36 \pm 0.08$ & $0.96 \pm 0.15$ & 7, possible:arc? \\
SDSS J162132.3+060719 & 0.343 & 7.4 & 16.2 & $19.88 \pm 0.06$ & $1.20 \pm 0.13$ & 7 , possible:giant arc? \\
SDSS J172336.1+341158 & 0.431 & $>8.5$ & 4.3 & $20.91 \pm 0.05$ & $-0.26 \pm 0.06$ & $7 *$, possible:multiple images? \\
\hline
\end{tabular}

Notes: Here we list the name, redshift and mass of cluster. The angular separation $(\theta)$ between the arc and the central galaxy, the $r$-band magnitude and color $(g-r)$ of the brightest part of arc, and also the references and notes on the lensing systems, are given in the following columns. References: (1) Estrada et al. (2007); (2) Shin et al. (2008); (3) Sand et al. (2005); (4) Lin et al. (2008); (5) Ofek et al. (2008); (6) Belokurov et al. (2009); (7) This work.

*: CASSOWARY candidates, see the end of Sect.3.

The total mass (including dark matter) within the Einstein ring $r_{\mathrm{E}}=D_{\mathrm{l}} \theta_{\mathrm{E}}$ can be estimated by,

$$
M\left(<r_{\mathrm{E}}\right)=\frac{c^{2} r_{\mathrm{E}}^{2}}{4 G} \frac{D_{\mathrm{s}}}{D_{\mathrm{l}} D_{\mathrm{ls}}}
$$

where $D_{\mathrm{s}}$ and $D_{\mathrm{l}}$ are the angular diameter distances of the source and lens from the observer, and $D_{\mathrm{ls}}$ is the angular diameter distance of the source from the lens. We estimate the angular Einstein ring $\theta_{\mathrm{E}}$ by $\theta$; this is an approximation due to the non-sphericity of clusters. In an $\Lambda \mathrm{CDM}$ cosmology $\left(\mathrm{H}_{0}=72\right.$ $\mathrm{km} \mathrm{s}^{-1} \mathrm{Mpc}^{-1}, \Omega_{m}=0.3$ and $\left.\Omega_{\Lambda}=0.7\right)$, we obtained the mass $M\left(<r_{\mathrm{E}}\right)=2.3 \times 10^{13} M_{\odot}$ for SDSS J090002.6+223404 if we assume the source redshift of $z_{\mathrm{s}}=1$, or $M\left(<r_{\mathrm{E}}\right)=1.6 \times 10^{13} M_{\odot}$ if $z_{\mathrm{s}}=2$. Similarly, for SDSS J223831.3+131955, the mass is $M\left(<r_{\mathrm{E}}\right)=2.3 \times 10^{13} M_{\odot}$ if $z_{\mathrm{s}}=1$ or $M\left(<r_{\mathrm{E}}\right)=1.7 \times 10^{13} M_{\odot}$ if $z_{\mathrm{s}}=2$.

We also found another 5 clusters which are probable lensing systems (see Fig. 2). All of them show blue arclets, which are tangential to the bright central galaxies and are distinct in color from the red cluster galaxies. Notably, the giant arcs in the clusters, SDSS J095739.1+050931 and NSCS $\mathrm{J} 122648.0+215157$, are faint, and have very large separations $\left(>10^{\prime \prime}\right)$ from the central galaxy. SDSS $\mathrm{J} 113740.0+493635$ has a blue arc, which is very close $\left(3.8^{\prime \prime}\right)$ to the central red galaxy. In the cluster SDSS maxBCG J120735.9+525459, the blue giant arc stands out among the red member galaxies.

Another 4 clusters are possible lensing systems (see Fig. 3). The blue images around the central galaxy of SDSS J123736.2+553342 may be the lensed image of a background source. However, this bright blue object on the top can also be a foreground galaxy. In the cluster SDSS J131534.2+233301, the three faint arclets may form a half ring surrounding several member galaxies rather than the brightest galaxy. These arclets may be independent, or they may just be faint member galaxies. In the cluster SDSS J162132.3+060719, the arc is tangential to the bright central galaxy and has a large separation $\left(16.2^{\prime \prime}\right)$, but does not have much color difference from the cluster galaxies. It may also be due to a combination of an edge-on galaxy plus other faint objects. The outstanding blue arclet in SDSS 

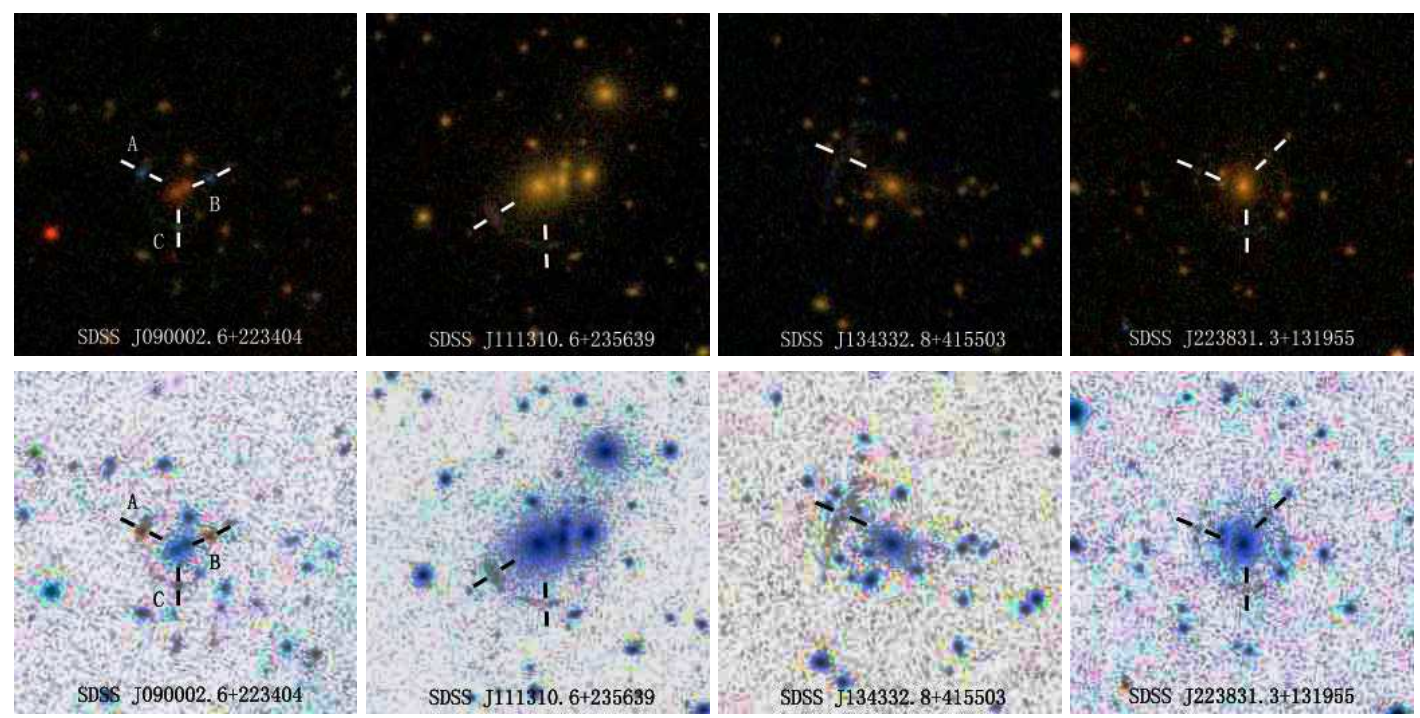

Fig. 1 The SDSS composite color images $(g, r$ and $i$ ) of 4 clusters which show Einstein rings or giant arcs. They are almost certain gravitational lensing systems. The images have a field of view of $1.2^{\prime} \times 1.2^{\prime}$. The negative images are also shown in the second rows to see more clearly the lensing features.

$\mathrm{J} 172336.1+341158$ is very close $\left(4.3^{\prime \prime}\right)$ to the central red galaxy. It may be the lensed and enhanced image of the background galaxy, but the possibility of the foreground galaxy can not be excluded.

\section{FINAL REMARKS}

Using the SDSS data, we almost certainly found 4 cluster lenses, plus 5 probable and 4 possible lenses. Together with 6 known lenses of clusters, there are at least 10 lensing systems identified from SDSS, which preferably have redshifts around 0.4 and masses of at least $5 \times 10^{14} M_{\odot}$. The separations of the blue arcs or rings from the central red galaxy are usually several arcseconds.

If the 19 clusters listed in Table 1 are all lenses, 6 of them have redshifts of $0.2<z<0.4$, and 13 of $0.4<z<0.6$. Comparing them with 7568 and 10121 clusters with masses $5 \times 10^{14} M_{\odot}$ in the corresponding redshift ranges (Wen et al. in preparation), we found that the occurrence probability of lensing clusters in the shallow SDSS images increases from $7.9 \times 10^{-4}$ in the range of $0.2<z<0.4$ to $1.3 \times 10^{-3}$ of $0.4<z<0.6$. The tendency of the increase in lensing probability with redshift is consistent with that of Gladders et al. (2003).

Follow-up observations are necessary to confirm the probable or possible lensing systems in 9 clusters. Unfortunately, we can not easily access large optical telescopes to make the follow-up confirmation. We also publish these candidates with a list and the SDSS color images to encourage followup observations by others. After we submitted this paper to this journal and to astro-ph, we learned from Dr. V. Belokurov that 5 objects with "*” in Table 1 have been listed in their CASSOWARY catalogue (see http://www.ast.cam.ac.uk/research/cassowary/) as lensing candidates CSWA 19, CSWA 10, CSWA 7, CSWA 13 and CSWA 14, respectively. The images of CSWA 7 and CSWA 13 have been published in Belokurov et al. (2009). These 5 objects therefore are independent "re-discoveries".

NOTES ADDED IN PROOF: 1) We found another probable lensing system by a merging cluster, NSCS J122648.0+215157 (Lopes et al. 2004), which we have added to Fig. 2 and Table 1, 2) We noted 

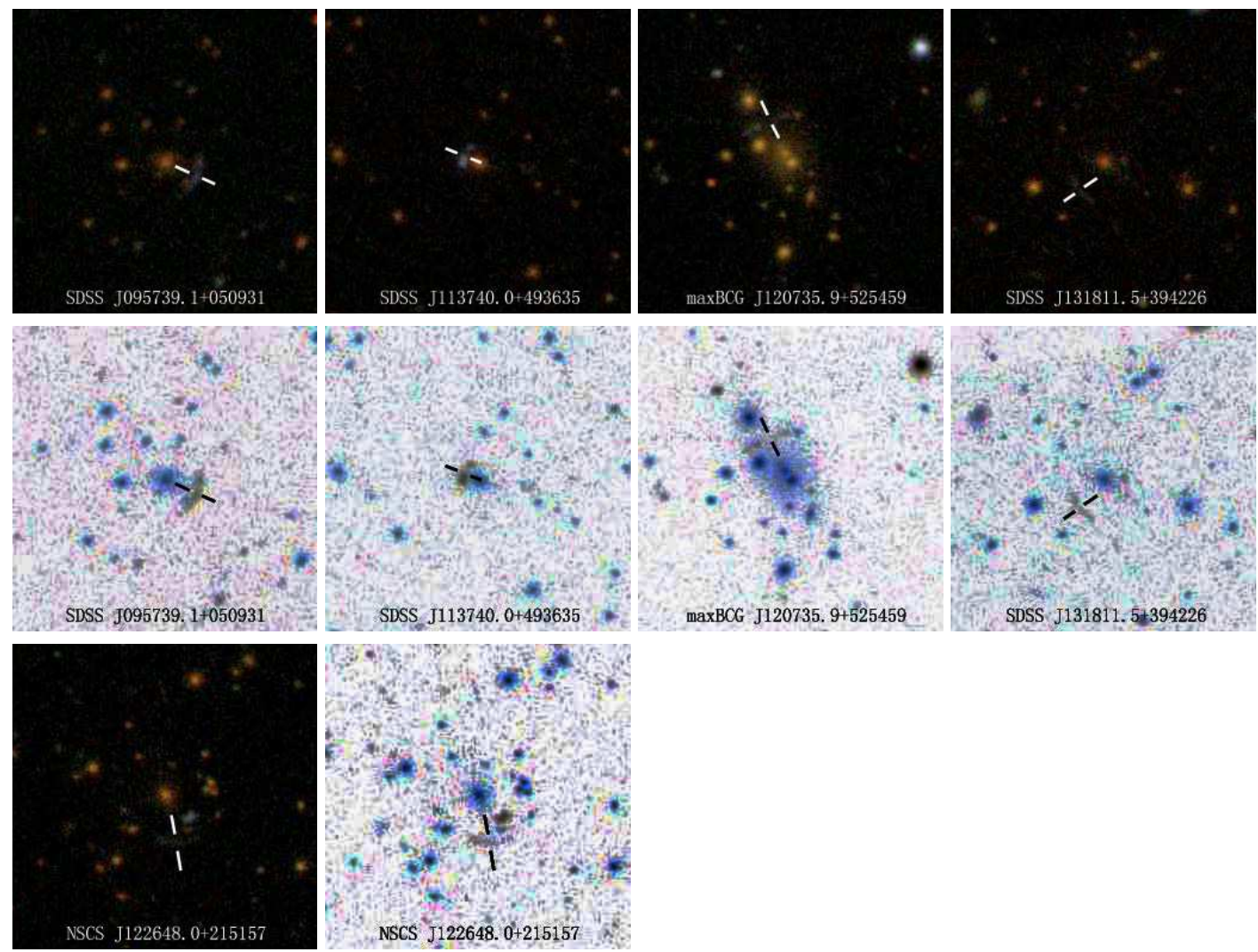

Fig. 2 Same as Fig. 1, but for 5 clusters which are probable lensing systems. The system of NSCS J122648.0+215157 in the last row was found from a merging cluster during the proof-reading stage of this paper.
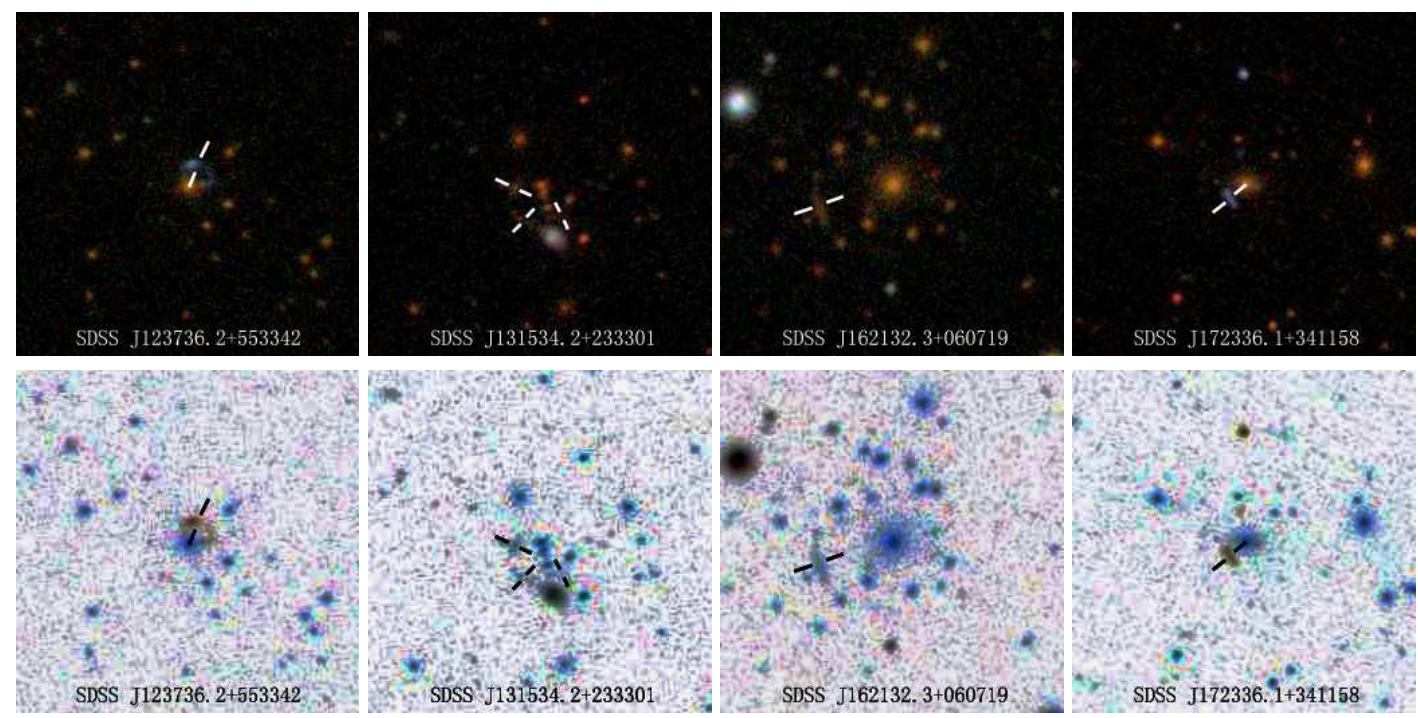

Fig. 3 Same as Fig. 1, but for 4 clusters which are possible lensing systems. 
that Kubo et al. (2008) just submitted a paper to report their SDSS arc survey results, including the follow-up observations of two lensing systems we found independently, SDSS J111310.6+235639 and J113740.0+493635.

Acknowledgements We thank Prof. Xiang-Ping Wu and Shude Mao for a carefully reading of the manuscript and the referee for helpful comments. The authors are supported by the National Natural Science Foundation of China (NSFC, Nos.10521001, 10773016 and 10833003) and the National Key Basic Research Science Foundation of China (2007CB815403). Funding for the SDSS and SDSS-II has been provided by the Alfred P. Sloan Foundation, the Participating Institutions, the National Science Foundation, the U.S. Department of Energy, the National Aeronautics and Space Administration, the Japanese Monbukagakusho, the Max Planck Society, and the Higher Education Funding Council for England.

\section{References}

Allam, S. S., Tucker, D. L., Lin, H., et al. 2007, ApJ, 662, L51

Bartelmann, M., Huss, A., Colberg, J. M., et al. 1998, A\&A, 330, 1

Belokurov, V., Evans, N. W., Moiseev, A., et al. 2007, ApJ, 671, L9

Belokurov, V., Evans, N. W., Hewett, P. C., et al. 2009, MNRAS, 392, 104

Csabai, I., Budavári, T., Connolly, A. J., et al. 2003, AJ, 125, 580

Estrada, J., Annis, J., Diehl, H. T., et al. 2007, ApJ, 660, 1176

Gladders, M. D., Hoekstra, H., Yee, H. K. C., et al. 2003, ApJ, 593, 48

Hennawi, J. F., Gladders, M. D., Oguri, M., et al. 2008, AJ, 135, 664

Koester, B. P., McKay, T. A., Annis, J., et al. 2007, ApJ, 660, 239

Kubo, J. M., Allam, S. S., Annis, J., et al. 2008, ApJ, submitted. (ArXiv e-print 0812.3934)

Li, G.-L., Mao, S., Jing, Y. P., et al. 2005, ApJ, 635, 795

Lin, H., Buckley-Geer, E., Allam, S. S., et al. 2008, ApJ, submitted. (ArXiv e-prints 0809.4475)

Lopes, P. A. A., de Carvalho, R. R., Gal, R. R., et al. 2004, AJ, 128, 1017

Luppino, G. A., Gioia, I. M., Hammer, F., et al. 1999, A\&AS, 136, 117

Lupton, R., Gunn, J. E., Ivezić, Z., et al. 2001, in Astronomical Society of the Pacific Conference Series, Vol. 238,

Astronomical Data Analysis Software and Systems X, ed. F. R. Harnden, Jr., F. A. Primini, \& H. E. Payne, 269 Lynds, R. \& Petrosian, V. 1989, ApJ, 336, 1

Metcalfe, L., Kneib, J.-P., McBreen, B., et al. 2003, A\&A, 407, 791

Miralda-Escude, J. \& Fort, B. 1993, ApJ, 417, L5

Ofek, E. O., Seitz, S., \& Klein, F. 2008, MNRAS, 389, 311

Reiprich, T. H. \& Böhringer, H. 2002, ApJ, 567, 716

Sand, D. J., Treu, T., Ellis, R. S., \& Smith, G. P. 2005, ApJ, 627, 32

Shin, M.-S., Strauss, M. A., Oguri, M., et al. 2008, AJ, 136, 44

Shu, C., Zhou, B., Bartelmann, M., et al. 2008, ApJ, 685, 70

Smail, I., Ellis, R. S., Fitchett, M. J., et al. 1991, MNRAS, 252, 19

Stoughton, C., Lupton, R. H., Bernardi, M., et al. 2002, AJ, 123, 485

Takahashi, R. \& Chiba, T. 2001, ApJ, 563, 489

York, D. G., Adelman, J., Anderson, Jr., J. E., et al. 2000, AJ, 120, 1579

Zaritsky, D. \& Gonzalez, A. H. 2003, ApJ, 584, 691 